\title{
Fatores relacionados a infecções de sítio cirúrgico após procedimentos obstétricos
}

\author{
Factors related to surgical site infections after obstetric procedures
}

\section{Catarina Escosteguy Petter ${ }^{1}$, Túlio Cícero Franco Farret ${ }^{1}$, Juliane de Souza Scherer ${ }^{2}$, Vicente Sperb Antonello ${ }^{3}$}

Médicos Residentes do Serviço de Ginecologia e Obstetrícia do Hospital Fêmina, Porto Alegre, RS.

2 Enfermeira do Serviço de Controle de Infecção Hospitalar do Hospital Fêmina, Porto Alegre, RS

Médico Infectologista do Serviço de Controle de Infecção Hospitalar do Hospital Fêmina, Porto Alegre, RS.

\begin{abstract}
RESUMO
Objetivos: A infecção de sítio cirúrgico representa a segunda maior causa de infecções hospitalares e uma das principais complicações no pós-parto. O presente estudo teve como objetivo descrever características e fatores de risco presentes em puérperas que tiveram infecção de sítio cirúrgico.

Métodos: Um estudo transversal retrospectivo foi realizado no Hospital Fêmina, incluindo os partos ocorridos de janeiro de 2009 a dezembro de 2010. De um total de 9528 partos, foram detectados e avaliados 110 prontuários de pacientes com diagnóstico de infecção de sítio cirúrgico após parto vaginal ou cesáreo.

Resultados: Foi encontrada taxa de infecção de sítio cirúrgico de 1,53\% para cesarianas e de 1,01\% para partos vaginais (risco relativo 1,5; IC 95\% 1,3-1,7). Entre as pacientes com infecção de sítio cirúrgico, muitas tinham baixo nível socioeconômico, eram obesas e não haviam sido submetidas a uma correta antibioticoprofilaxia no pré-parto.

Conclusões: O risco de desenvolver infecção foi uma vez e meia maior após um parto cesáreo do que após um parto vaginal. Os índices de infecção de sítio cirúrgico pós-parto encontrados neste estudo, assim como algumas características das pacientes que desenvolveram infecções, reforçam a importância de identificar possíveis fatores de risco presentes e de buscar a prevenção nas pacientes obstétricas com a normatização de condutas, incluindo o uso correto de antibioticoterapia profilática.
\end{abstract}

DESCRITORES: INFECÇÃO DA FERIDA OPERATÓRIA; PUERPÉRIO; INFECÇÃO PUERPERAL; PARTO OBSTÉTRICO; FATORES DE RISCO; CESÁREA; PARTO NORMAL.

\begin{abstract}
Aims: Surgical site infection is the second main cause of nosocomial infections and a major complication during postpartum period. This study aimed to describe characteristics and risk factors found in puerperal women who had surgical site infection.

Methods: A retrospective cross-sectional study was conducted at Hospital Femina, Porto Alegre, Rio Grande do Sul, Brazil, including deliveries from January 2009 to December 2010. From a total of 9528 deliveries, 110 records of patients diagnosed with surgical site infection after vaginal delivery or cesarean section were detected and analyzed. Results: We found a rate of surgical site infection of $1.53 \%$ for cesarean deliveries and of $1.01 \%$ for vaginal deliveries (RR 1.5, 95\%CI 1.3-1.7). Among the patients with surgical site infection many had low socioeconomic status, were obese and had incorrect administration of antibiotic prophylaxis in pre-partum.

Conclusions: The risk of infection was one and a half times greater after a caesarean section than after a vaginal delivery. The rates of postpartum surgical site infection found in this study, as well as some characteristics of the patients who developed infections, stress the importance of identifying potential risk factors and to seek prevention in obstetric patients through standards of practice that include proper use of prophylactic antibiotics.
\end{abstract}

KEY WORDS: SURGICAL WOUND INFECTION; PUERPERIUM; PUERPERAL INFECTION; DELIVERY, OBSTETRIC; RISK FACTORS; CESAREAN SECTION; NATURAL CHILDBIRTH. 


\section{INTRODUÇÃO}

As infecções hospitalares representam importante problema de saúde pública por aumentarem a morbidade e a mortalidade dos pacientes, além dos custos hospitalares. ${ }^{1,2}$ Infecção hospitalar é definida como aquela adquirida após a admissão do paciente e que se manifesta durante a internação ou após a alta, relacionada com o hospital ou com procedimentos hospitalares. ${ }^{1,3}$

Infecção de sítio cirúrgico (ISC) representa a segunda maior causa de infecção hospitalar. Entre os pacientes cirúrgicos, ISC é a infecção mais frequente, correspondendo a $38 \%$ do total das infecções hospitalares. - $^{4-6}$ Estima-se que a ISC prolongue o tempo de internação em mais de sete dias e aumente o risco de reinternação em 15 vezes, causando repercussões para os pacientes e o hospital. ${ }^{6,7}$ No Brasil, há descrição de ocorrência de ISC em 2,8 a $20 \%$ das cirurgias, com média de $11 \%$, dependendo do tipo de vigilância realizada, das características do hospital, do paciente e do tipo de procedimento cirúrgico. ${ }^{8} 9$ Nos Estados Unidos da América a incidência estimada é de 2 a 5\%, representando mais de 500.000 novos casos por ano, podendo variar entre 2 a $5 \%$ para cirurgias limpas e mais de $40 \%$ para algumas cirurgias contaminadas. ${ }^{10-13}$

Múltiplos fatores podem contribuir para o aumento do risco de ISC. Dos fatores relacionados ao paciente, muitos não podem ser modificados. Como exemplos de fatores de risco podem-se citar extremos de idade, estado nutricional prejudicado, diabetes, obesidade, tabagismo, imunossupressão, infecção em outro sítio e má higiene cutânea. ${ }^{2,11}$ Entre as características perioperatórias incluem-se, entre outras, técnica cirúrgica inadequada, tempo cirúrgico maior que duas horas, perda sanguínea excessiva, tempo prolongado de internação pré-operatória e uso inadequado de antibioticoprofilaxia prévia à cirurgia. ${ }^{14-17}$

Do ponto de vista obstétrico, a infecção puerperal representa uma das principais complicações em sítio cirúrgico no pós-parto. Pode ocorrer em sítio cirúrgico incisional (ferida operatória ou episiotomia) e em cavidade uterina (endometrite). Trabalho de parto prolongado, ruptura prematura de membranas, excesso na manipulação vaginal, grande quantidade de mecônio no líquido amniótico, extração manual da placenta e parto prematuro podem aumentar o risco de ISC obstétrico. ${ }^{2,4,15,18}$ Comorbidades, como doença pelo HIV, anemia severa e diabetes gestacional, estão associadas a maiores índices de infecção puerperal. ${ }^{19,20}$

Parto cesáreo é o fator de risco mais importante para o desenvolvimento da infecção puerperal. ${ }^{17} \mathrm{Na}$ ausência de profilaxia antibiótica, as taxas de endometrite são de aproximadamente $30 \%$ após cesárea de urgência, $7 \%$ após cesárea eletiva e menos de $3 \%$ após parto vaginal. $^{21,22}$ É importante destacar que a profilaxia antimicrobiana antes do parto cesáreo, administrada corretamente, diminui o risco de endometrite e ISC incisional em 40 e $30 \%$, respectivamente. ${ }^{23}$

$\mathrm{O}$ presente estudo pretende descrever fatores de risco encontrados em puérperas que apresentaram ISC e comparar os grupos de parto cesáreo e parto vaginal, relacionando suas variáveis demográficas e clínicas.

\section{MÉTODOS}

Foi realizado um estudo transversal, retrospectivo e descritivo, no Hospital Fêmina, instituição referência na cidade de Porto Alegre (capital do estado do Rio Grande do Sul) e sua região metropolitana, para o atendimento às mulheres durante o pré-natal, parto e puerpério. O estudo foi aprovado pelo Comitê em Ética em Pesquisa do Grupo Hospitalar Conceição, em 11 de abril de 2011 com o número de registro 11-023.

Foram avaliados prontuários de pacientes com diagnóstico de ISC após procedimentos obstétricos, de janeiro de 2009 a dezembro de 2010 . Incluiram-se as pacientes com endometrite, infecção de ferida operatória ou infeção de episiotomia, de acordo com os critérios da National Nosocomial Infections Surveillance System (NNIS) recomendados pela Agência Nacional de Vigilância Sanitária (ANVISA). ${ }^{24}$ As pacientes foram identificadas a partir da comunicação regular sobre infecções e da notificação do Serviço de Controle de Infecção Hospitalar do Hospital Fêmina. Foram avaliados dados demográficos das pacientes e fatores de risco.

O risco cirúrgico pré-operatório foi classificado por intermédio do escore ASA (American Society of Anestesiologists), que avalia a condição clínica pré-operatória do paciente. ${ }^{25}$ ASA 1: sem distúrbios fisiológicos, bioquímicos ou psiquiátricos; ASA 2: distúrbio fisiológico leve a moderado, controlado, sem comprometimento da atividade normal; ASA 3: distúrbio sistêmico importante, de difícil controle, com comprometimento da atividade normal e com impacto sobre a anestesia e a cirurgia; ASA 4: distúrbio sistêmico severo, potencialmente letal, com grande impacto sobre a anestesia e a cirurgia; ASA 5: paciente moribundo. Pacientes com escores ASA de 1 a 2 são considerados de baixo risco para complicações cirúrgicas e com ASA maior ou igual a 3 têm alto risco de complicações cirúrgicas.

Para a análise estatística foi utilizada avaliação descritiva com média e desvio padrão para variáveis contínuas e frequência e percentagem para variáveis 
categóricas. Na comparação para as médias entre grupos com ISC utilizamos o teste $\mathrm{t}$ de Student e, para análise das frequências, o teste qui-quadrado. Para avaliar o risco comparativo de desenvolvimento de ISC entre os grupos de parto cesáreo e vaginal, utilizamos risco relativo (RR) e intervalo de confiança (IC) de 95\%. Foi utilizado como banco de dados o programa Microsoft Excel e, para análise, o programa SPSS (Statistical Package for Social Sciences) para Windows.

\section{RESULTADOS}

De um total de 9528 partos (4106 partos cesáreos e 5422 partos normais), foram notificados 118 $(1,23 \%)$ casos de ISC. Encontramos $1,53 \%$ de ISC para cesárea e $1,01 \%$ para parto vaginal espontâneo. Assim, nas pacientes que tiveram parto cesáreo o risco de desenvolver ISC foi 1,5 vezes maior do que nas pacientes com parto vaginal (RR 1,5; IC 95\% 1,3-1,7; $\mathrm{p}=0,03)$.

Os prontuários referentes a 111 partos $(94 \%$ dos casos de ISC) estiveram disponíveis para análise, sendo 59 partos cesáreos e 52 partos vaginais. A média de idade do grupo total de pacientes avaliadas foi de 24,1 anos. Apenas três pacientes tinham mais de 35 anos. Nenhuma paciente possuía ensino superior, 73 pacientes tinham cursado o primeiro grau de ensino, 36 o segundo grau e apenas uma paciente era analfabeta. Em 63 pacientes $(57 \%)$ pôde-se obter peso e altura. Entre estas, $52(82,5 \%)$ tinham índice de massa corporal (IMC) $>25 \mathrm{~kg} / \mathrm{m}^{2}$ e $29(46 \%)$ eram obesas.

Em relação ao risco cirúrgico (escore ASA), comorbidades, complicações obstétricas, tempo de internação hospitalar e paridade, os dados encontram-se na Tabela 1. A medida de hemoglobina antes do parto esteve disponível em 61 pacientes (55\%), variando entre 8 e 13,6 mg/dl, com média de $11,29 \mathrm{mg} / \mathrm{dl}$.

O tempo de hospitalização pré-operatória variou de 0 a 6 dias, sendo que em 100 pacientes (91\%) o procedimento ocorreu em um dia ou menos. Quanto ao tempo total de internação hospitalar, a média foi de $5,65 \pm 4,6$ dias. Oito pacientes haviam tido internação anterior recente (há menos de um mês antes da internação para o parto).

O número de consultas de pré-natal era disponível para 100 pacientes, dentre as quais $66(66 \%)$ tiveram seis ou mais consultas. Nenhum procedimento (parto vaginal ou cesárea) teve duração maior que uma hora e meia. A média da idade gestacional das pacientes no momento da interrupção da gestação foi de $38 \pm 3$ semanas.
Quando classificado o grupo com ISC de acordo com a via de parto (cesárea ou vaginal) observamos que no grupo de parto cesáreo as pacientes tinham idade maior, tempo de internação mais prolongado e maior número de toques vaginais. A diferença no IMC e em outros dados levantados não foi estatisticamente significativa (Tabela 2 ).

Tabela 1. Características das pacientes que tiveram infecção de sítio cirúrgico após procedimentos obstétricos (parto cesáreo e parto vaginal) no Hospital Fêmina, Porto Alegre, RS, 2009-2010.

\begin{tabular}{lcc}
\hline \multicolumn{1}{c}{ Variáveis } & $\mathbf{n}$ & $\mathbf{\%}$ \\
\hline Total de prontuários estudados & $\mathbf{1 1 1}$ & $\mathbf{1 0 0}$ \\
ASA 1 & 101 & 91 \\
ASA 2 & 5 & 4.5 \\
ASA 3 & 5 & 4.5 \\
Comorbidades & & \\
Hipertensão arterial sistêmica & 7 & 6.3 \\
Infecção por HIV & 3 & 2.7 \\
Sífilis & 2 & 1.8 \\
Tabagismo & 12 & 11 \\
Complicações & & \\
Infecção de trato urinário & 13 & 12 \\
Pré-eclâmpsia & 9 & 8 \\
RUPREMA & 6 & 5.5 \\
Tempo de hospitalização & & \\
2-3 dias & 51 & 46 \\
4-10 dias & 43 & 39 \\
> 10 dias & 16 & 15 \\
Paridade & & \\
Primeira gestação & 55 & 50 \\
Segunda gestação & 36 & 33 \\
Terceira gestação ou posterior & 19 & 17 \\
\hline
\end{tabular}

ASA: escore da American Society of Anestesiologists. ASA 1: sem distúrbios fisiológicos, bioquímicos ou psiquiátricos; ASA 2: distúrbio fisiológico leve moderado, controlado, sem comprometimento da atividade normal; ASA 3: distúrbio sistêmico importante, de difícil controle, com comprometimento da atividade normal e com impacto sobre a anestesia e a cirurgia.

RUPREMA: ruptura prematura de membranas amnióticas.

Tabela 2. Características das pacientes que tiveram infecção de sítio cirúrgico após procedimentos obstétricos no Hospital Fêmina, Porto Alegre, RS, 2009-2010, conforme a via de parto (cesárea ou parto vaginal).

\begin{tabular}{lccc}
\hline & $\begin{array}{c}\text { Parto cesáreo } \\
(\mathbf{n = 5 9 )}\end{array}$ & $\begin{array}{c}\text { Parto vaginal } \\
(\mathbf{n}=\mathbf{5 2})\end{array}$ & $\mathbf{P}$ \\
\hline $\begin{array}{lccc}\text { Idade em anos } \\
\text { (média } \pm \text { desvio padrão) }\end{array}$ & $25,86 \pm 6,62$ & $22,17 \pm 5,57$ & 0,001 \\
Caucasianas (\%) & $82 \%$ & $78 \%$ & $>0,05$ \\
IMC (kg) & $32,66 \pm 9,09$ & $28,90 \pm 5,4$ & 0,065 \\
Tempo de internação (dias) & $6,74 \pm 5,14$ & $4,36 \pm 3,4$ & 0,004 \\
$\begin{array}{l}\text { Hb prévia ao procedimento } \\
\text { (g/dl) }\end{array}$ & $11,5 \pm 1,14$ & $10,9 \pm 1,49$ & 0,09 \\
$\begin{array}{l}\text { Número de consultas de } \\
\text { pré-natal }\end{array}$ & $6,43 \pm 3,32$ & $6,22 \pm 2,74$ & 0,73 \\
Número de toques vaginais & $3,76 \pm 2,48$ & $5,63 \pm 1,88$ & $<0,01$ \\
\hline
\end{tabular}

IMC: Índice de massa corporal; Hb: Hemoglobina; 
Avaliou-se a profilaxia antimicrobiana antes do parto cesáreo. Dos 59 partos cesáreos, em nove não há descrição de profilaxia, e em seis casos não há descrição da hora da administração. Em 44 casos pôdese avaliar o regime de profilaxia antimicrobiana: em cinco casos (11\%) a profilaxia foi feita 30-60 minutos antes do procedimento; em 36 casos (82\%) foi feita após o início do procedimento; e em três casos (7\%) realizada a menos de 30 minutos antes do parto.

\section{DISCUSSÃO}

Índices de ISC descritos na literatura após cesariana variam de 3 a $15 \% .{ }^{26-28}$ Yokoe et al., ${ }^{29} \mathrm{em}$ estudo publicado em 2001, encontraram 4,2\% de ISC após cesárea e $0,5 \%$ após parto vaginal no Brigham and Women's Hospital, na área de Boston, Estados Unidos. Allen et al., ${ }^{30} \mathrm{em}$ um estudo de coorte de base populacional conduzido na Nova Escócia, Canadá, entre $1988-2001$, encontraram $1,95 \%$ e $0,77 \%$ de incidência de ISC para parto cesáreo e vaginal, respectivamente.

Dados americanos do National Healthcare Safety Network, ${ }^{31}$ publicados em 2009, mostraram média para ISC em cesáreas de $1,46 \%$, semelhante ao encontrado no presente estudo, com $1,53 \%$ de ISC para cesárea e $1,01 \%$ para parto vaginal espontâneo.

Autores consideram como fatores de risco para ISC idade materna acima de $35 \operatorname{anos}^{32}$ e primiparidade. ${ }^{33} \mathrm{~A}$ média de idade das pacientes no presente estudo foi de 24 anos, e 50\% delas eram primíparas. Somente três pacientes tinham mais de 35 anos. Segundo o Ministério da Saúde, o número de consultas de pré-natal deve ser no mínimo seis para que o acompanhamento seja considerado adequado. ${ }^{34}$ Em nossa amostra, somente $66 \%$ das pacientes tiveram seis consultas ou mais. Algumas comorbidades estão associadas ao risco de endometrite puerperal, como síndrome da imunodeficiência adquirida e diabetes. ${ }^{19,32,35}$ As comorbidades mais prevalentes em nosso estudo foram infecção pelo HIV, sífilis e hipertensão. Entretanto, $91 \%$ das pacientes não apresentavam quaisquer comorbidades.

A obesidade está bem documentada na literatura como fator de risco para infecção pós-operatória. ${ }^{26,36}$ Magann et al. ${ }^{37}$ observaram que mulheres com IMC maior do que 30 são mais propensas a desenvolver infecção de ferida operatória do que mulheres com IMC normal. No presente estudo, a grande maioria das pacientes $(82,5 \%)$ tinha sobrepeso, sendo que $46 \%$ eram obesas.

Quanto ao risco cirúrgico, mais de $90 \%$ das pacientes no presente estudo apresentaram ASA 1, provavelmente devido à média de idade das gestantes, menor do que 25 anos. A probabilidade de infecção em cirurgias não contaminadas e classificadas como ASA 1, descrita na literatura, é de 2,2\%. ${ }^{37}$ Adicionalmente, o tabagismo foi encontrado em $11 \%$ das pacientes. Sabe-se que o tabaco dificulta a cicatrização o que torna a ferida cirúrgica mais susceptível à infecção. ${ }^{2}$

O tempo de hospitalização pré-operatório maior que sete dias tem sido associado com o desenvolvimento de infecção de sítio cirúrgico. ${ }^{15-17} \mathrm{Em} 91 \%$ da nossa amostra o procedimento obstétrico ocorreu em um dia ou menos de internação. O tempo cirúrgico prolongado (maior do que duas horas) também é um importante fator de risco para ISC. ${ }^{14,17}$ No nosso estudo nenhum procedimento excedeu uma hora e meia de duração.

Em relação à profilaxia pré-operatória, a prática obstétrica usual em alguns serviços quanto ao momento da realização do antibiótico preventivo nas cesáreas tem sido de administrar a medicação após o clampeamento do cordão umbilical. ${ }^{38}$ Porém, estudos vêm questionando esta prática. Owens et al..$^{23}$ demonstraram que a administração antibiótica profilática 60 minutos antes da incisão da pele, comparada com o uso do antibiótico após o clampeamento do cordão umbilical, foi associada à redução de $40 \%$ na endometrite pósparto e de $30 \%$ na ISC. Em nosso estudo, em $82 \%$ dos 44 casos nos quais se pôde avaliar o momento exato da profilaxia, a medicação foi feita após o início do procedimento.

Uma metanálise que avaliou cinco estudos, entre eles três ensaios clínicos randomizados, encontrou evidências de que o uso do antibiótico profilático em cesarianas realizado antes da incisão da pele diminui a incidência de endometrite pós-parto e morbidade infecciosa, sem afetar os resultados neonatais. ${ }^{39}$ A recomendação na literatura é a administração do antibiótico profilático 60 minutos antes da incisão cirúrgica, resultando em níveis terapêuticos na ferida cirúrgica e tecidos adjacentes durante $\mathrm{o}$ procedimento. ${ }^{17,40}$ Passados os 60 minutos antes do procedimento, a administração da profilaxia 30 a 60 minutos antes da cirurgia parece ser mais eficaz do que a administração imediatamente antes do procedimento. ${ }^{40}$ Nesta amostra, em somente $11 \%$ das pacientes foi feita a profilaxia entre 30 e 60 minutos.

Alguns autores consideram que o parto cesáreo causa de 5 a 30 vezes maior risco de infecção do que o parto vaginal..$^{2,30,32} \mathrm{De}$ acordo com os resultados do presente estudo, o risco de desenvolver ISC foi 1,5 vezes maior nas pacientes do grupo cesárea do que nas pacientes do grupo parto vaginal. 
A incidência de nascimentos por cesariana tem aumentado significativamente nos últimos anos, demonstrando índices maiores do que o recomendado pela Organização Mundial da Saúde. No Sistema Único de Saúde as cesáreas somam $26 \%$ do total de partos, quando o recomendado seria no máximo $15 \% .^{41}$ No presente estudo encontramos $43 \%$ dos nascimentos ocorridos por cesárea e $57 \%$ por parto normal.

Como limitações deste estudo, a principal é ter sido uma pesquisa retrospectiva de prontuários. A escassez de informações nos prontuários de algumas pacientes pode ter prejudicado a análise completa dos fatores de risco. A falta de um grupo controle sem infecção não nos permitiu avaliar as associações com fatores de risco.

Os índices de ISC após parto vaginal e cesáreo encontrados reforçam a importância da identificação dos fatores de risco envolvidos e da busca por prevenção de infecção puerperal em pacientes obstétricas, com a normatização de condutas, como, por exemplo, o uso adequado da antibioticoterapia profilática. Após a análise dos dados deste estudo foi inserido um protocolo de realização de antibioticoterapia profilática entre 30-60 minutos antes das cesarianas no Hospital Fêmina. Finalmente, é necessária a realização de estudos comparativos com grupo controle e estudos longitudinais prospectivos sobre o assunto, para maiores esclarecimentos e avaliação dos fatores de risco, assim como para detectar possíveis mudanças no perfil epidemiológico das pacientes com ISC.

\section{AGRADECIMENTOS}

Agradecemos a todos os membros do Serviço de Controle de Infecção Hospitalar do Hospital Fêmina pelo apoio e colaboração no presente trabalho.

\section{REFERÊNCIAS}

1. McKibben L, Horan TC, Tokars JI, et al. Guidance on public reporting of healthcare-associated infections: recommendations of the Healthcare Infection Controle Practices Advisory Comitee. Infect Control Hosp Epidemiol. 2005;26:580-7.

2. Talbot TR. Surgical site infections and antimicrobial prophylaxis. In: Mandell GL, Bennett JE, Dolin R. Mandell, Douglas and Bennett's principles and practice of infectious diseases. $7^{\text {th }}$ ed. New York: Churchill Livingstone; 2009.

3. Garner JS, Jarvis WR, Emori TG, et al. CDC definition for nosocomial infections. Am J Infect Control. 1988;16: 128-40.

4. Consensus paper on the surveillance of surgical wound infections. The Society for Hospital Epidemiology of America; The Association for Practitioners in Infection Control; The Centers for Disease Control; The Surgical Infection Society. Infect Control Hosp Epidemiol. 1992;13:599-605.
5. Horan TC, Culver DH, Gaynes RP, et al. Nosocomial infections in surgical patients in the United States, January 1986-June 1992. National Nosocomial Infections Surveillance (NNIS) System. Infect Control Hosp Epidemiol. 1993;14:73-80.

6. Oliveira CO, Ciosak SI. Infection of surgical site in the following postdischarge: impact in the incidence and evaluation of the used methods. Rev Esc Enferm USP. 2004;38:379-85.

7. Kirkland KB, Briggs JP, Trivette SL, et al. The impact of surgical-site infections in the 1990s: attributable mortality, excess length of hospitalization, and extra costs. Infect Control Hosp Epidemiol. 1999;20:725-30.

8. Ferraz EM, Bacelar TS, Aguiar JLA. Controle de infecção em cirurgia geral: resultados de um estudo prospectivo de 13 anos e de 17503 operações. Rev Col Bras Cir. 1992;19: 169-74.

9. Ferraz EM, Ferraz AAB, Coelho HSTA, et al. Postdischarge surveillance for nosocomial infection: does judicious monitoring find cases? Am J Infect Control. 1995;23:290-4.

10. Cruse P. Wound infection surveillance. Rev Infect Dis. 1981;3:734-7.

11. Anderson DJ, Kaye KS, Classen D, et al. Strategies to prevent surgical site infections in acute care hospitals. Infect Control Hosp Epidemiol. 2008;29 Suppl 1:S51-61.

12. Giribella AHG, Neme RM, Margarido PF, et al. Infecção da ferida cirúrgica em ginecologia. Rev Ginec \& Obst. 1999; 10:157-162.

13. Melling AC, Ali B, Scott EM, et al. Effects os preoperative warming on the incidence of wound infection after clean surgery: a randomized controlled trial. Lancet. 2001; 358:876-80.

14. Haley RW, Culver DH, Morgan WM, et al. Identifying patients at high risk of surgical wound infection. A simple multivariate index of patient susceptibility and wound contamination. Am J Epidemiol. 1985;121:206-15.

15. Oliveira JCC, Blank N, Damerau EF. Fatores de risco para infecção de sítio cirúrgico em cirurgia colorretal eletiva. Rev Bras Coloproct. 2001;21:75-83.

16. Mangram AJ, Horan TC, Pearson ML, et al. Guideline for prevention of surgical site infection, 1999. Hospital Infection Control Practices Advisory Committee. Infect Control Hosp Epidemiol. 1999;20:250-78.

17. Bratzler DW, Hunt DR. The surgical infection prevention and surgical care improvement projects: national initiatives to improve outcomes for patients having surgery. Clin Infect Dis. 2006; 43:322-30.

18. Wilkinson C, Enkin MW. Manual removal of placenta at caesarean section. Cochrane Database Syst Rev. 2000; CD000130.

19. Diamond MP, Entman SS, Salyer SL, et al. Increased risk of endometritis and wound infection after cesarean section in insulin-dependent diabetic women. Am J Obstet Gynecol. 1986;155:297-300.

20. Louis J, Buhari MA, Allen D, et al. Incidence of postcesarean infections in relation to HIV status in a setting with limited resources. Acta Obstet Gynecol Scand. 2005;84:967-71.

21. Burrows LJ, Meyn LA, Weber AM. Maternal morbidity associated with vaginal versus cesarean delivery. Obstet Gynecol. 2004;103(5 Pt 1):907-12.

22. Smaill F, Hofmeyr GJ. Antibiotic prophylaxis for cesarean section. Cochrane Database Syst Rev. 2000; CD000933.

23. Owens SM, Brozanski BS, Meyn LA, et al. Antimicrobial prophylaxis for cesarean delivery before skin incision. Obstet Gynecol. 2009;114:573-9. 
24. Agência Nacional de Vigilância Sanitária. Sítio cirúrgico: critérios nacionais de infecções relacionadas à assistência à saúde. 2009. [Acesso em 19 de novembro de 2011]. Disponível em: http:/www.anvisa.gov.br/servicosaude/ manuais/criterios_nacionais_ISC.pdf

25. The American Society of Anesthesiologists. ASA Physical Status Classification System. [Internet]. [capturado 2013 jan 17]:[1 tela]. Disponível em http://www.asahq.org/Home/ For-Members/Clinical-Information/ASA-Physical-StatusClassification-System

26. Olsen M, Butler AM, Willers DM, et al. Risk Factors for surgical site infection after low transverse cesaren section. Infect Control Hosp Epidemiol. 2008;29:477-84.

27. Chaim W, Bashiri A, Bar-David J, et al. Prevalence and clinical significance of postpartum endometritis and wound infection. Infect Dis Obstet Gynecol. 2000;8:77-82.

28. Mitt P, Lang K, Peri A, et al. Surgical-site infections following cesarean section in an Estonian university hospital: postdischarge surveillance and analysis of risk factors. Infect Control Hosp Epidemiol. 2005;26:449-54.

29. Yokoe DS, Christiansen CL, Johnson R, et al. Epidemiology of and surveillance for postpartum infections. Emerg Infect Dis. 2001;7:837-41.

30. Allen VM, O'Conell CM, Liston RM, et al. Maternal morbidity associated with cesarean delivery without labor compared with spontaneous onset of labor at term. Obstet Gynecol. 2003;102:477-82.

31. Edwards JR, Peterson KD, Mu Y, et al. National Healthcare Safety Network (NHSN) report: data summary for 2006 through 2008, issued December 2009. Am J Infect Control. 2009; 37: 783-805.
32. Capuzzi IF, Pereira AH, Silveira C. Análise dos fatores de risco em puérperas com infecção de sítio cirúrgico em unidade hospitalar de obstetrícia. Perspect Med. 2007; 18:11-6.

33. Maharaj D. Puerperal pyrexia: a review. Part II. Obstet Gynecol Surv. 2007;62:400-6.

34. Ministério da Saúde. Secretaria Executiva. Programa Humanização do Parto: humanização no pré-natal e nascimento. Brasília: Ministério da Saúde; 2002.

35. Louis J, Buhari MA, Allen D, et al. Postpartum morbidity associated with advanced HIV disease. Infect Dis Obstet Gynecol. 2006;2006:79512.

36. Magann EF, Doberty DA, Chauban SP, et al. Pregnancy, obesity, gestational weight gain, and parity as predictors of peeripartum complications. Arch Gynecol Obstet. 2011; 284:827-36.

37. Garibaldi RA, Cushing O, Lerer T. Risk factors for postoperative infection. Am J Med 1991;91:158S-162S.

38. Freitas F, Martins-Costa SH, Ramos JGL, et al. Rotinas em obstetrícia. $6^{\mathrm{a}}$ ed. Porto Alegre: Artmed; 2011.

39. Constantine MM, Raham M, Ghulmiyah L, et al. Timing of perioperative antibiotics for cesarean delivery: a metaanalysis. Am J Obstet Gynecol. 2008;199:301.e1-6.

40. Weber WP, Marti WR, Zwahlen M, et al. The timing of surgical antimicrobial prophylaxis. Ann Surg. 2008;247: 918-26.

41. Villar J, Valladares E, Wojdyla D, et al. Caesarean delivery rates and pregnancy outcomes: the 2005 WHO global survey on maternal and perinatal health in Latin America. Lancet. 2006;367:1819-29. 